\title{
SMYD2 in Leukemia: An Update
}

\section{Teresa Rubio-Tomás ${ }^{1,2^{*}}$ (iD}

${ }^{1}$ Institut d'Investigacions Biomèdiques August Pi i Sunyer (IDIBAPS), Barcelona, Spain

${ }^{2}$ School of Medicine, University of Crete, Crete, Greece

*Corresponding author: Teresa Rubio-Tomás, Institut d'Investigacions Biomèdiques August Pi i Sunyer (IDIBAPS), Barcelona, Spain; School of Medicine, University of Crete, Crete, Greece

\begin{abstract}
SMYD2 is one of the five members (SMYD1-5) of the Su(Var)3-9, Enhancer-of-zeste and Trithorax (SET) and Myeloid, Nervy, and DEAF-1 (MYND) domain-containing (SMYD) protein family and is it known to methylate histone and non-histone substrates. By methylating a wide range of targets, SMYD2 acts as an oncogene in most cancer types.

In this review I will comment on the last publications related to the role of SMYD2 in leukemia and I will refer to more extensive reviews if the reader aims to have a broader picture of the state of the art.
\end{abstract}

\section{SMYD2 Promotes Leukemia Progression}

Remarkably, SMYD2 is not only involved in leukemia, but also normal lineage differentiation of hematopoietic stem cells, since mice lacking SMYD2 specifically in hematopoietic stem cells displayed aberrant lymphocyte development. Regarding leukemia, these SMYD2 knockout mice had a high rate of apoptosis and showed loss of anchorage-independent transformation of leukemia cells [1]. In line with these observations, the authors detected overexpression of SMYD2 in many types of human leukemia [1]. Even residual expression of SMYD2 (and SMYD3) can promote chronic lymphocytic leukemia, probably due to the acquisition of complex karyotype [2].

Zipin-Roitman, et al. observed that decreased expression of SMYD2 leads to overexpression of SET7/9, indicating some kind of interplay between these two methyl transferases, that results in higher resistance to DNA damage of leukemia cells [3]. In addition, SMYD2 seemed to be downstream of MYC in acute myeloid leukemia [4].
With respect to pediatric leukemia, clinical studies indicate that upregulation of SMYD2 mRNA levels is associated with poor prognosis (i.e. higher white blood cell count, lower overall and event-free survival, among other parameters analyzed) in pediatric B lineage acute lymphoblastic leukemia, whereas SMYD2 expression was downregulated after remission of the disease [5]. Similar results were obtained by Sakamoto, et al. in the same pediatric disease [6].

\section{Conclusions and Further Directions}

Despite that the relationship between SMYD2 and tumorigenesis has been widely studied [7] and specific SMYD2 inhibitors exist (although they are not yet used in the clinics) [8], further studies are needed to elucidate its role in leukemia, as well as in other aspects of immunology [9].

\section{References}

1. Brown MA, Edwards MA, Alshiraihi I, Geng $H$, Dekker JD, et al. (2020) The lysine methyltransferase SMYD2 is required for normal lymphocyte development and survival of hematopoietic leukemias. Genes Immun 21: 119-130.

2. Oliveira-Santos W, Rabello DA, Lucena-Araujo AR, de Oliveira FM, Rego EM, et al. (2016) Residual expression of SMYD2 and SMYD3 is associated with the acquisition of complex karyotype in chronic lymphocytic leukemia. Tumor Biology 37: 9473-9481.

3. Adi Z-R, Aqaqe N, Yassin M, Biechonski S, Amar M, et al. (2017) SMYD2 lysine methyltransferase regulates leukemia cell growth and regeneration after genotoxic stress. Oncotarget 8: 16712-16727.

4. Sevgi B, Sabo A, Kress TR, Doni M, Nicoli P, et al. (2016) Smyd2 is a Myc-regulated gene critical for MLL-AF9 induced leukemogenesis. Oncotarget 7: 66398-66415.

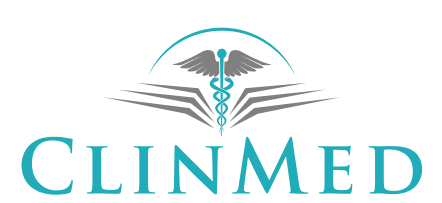

INTERNATIONAL LIBRARY
Citation: Tomás TR (2021) SMYD2 in Leukemia: An Update. Int J Blood Res Disord 8:067. doi. org/10.23937/2469-5696/1410067

Accepted: August 28, 2021: Published: August 30, 2021

Copyright: @ 2021 Tomás TR. This is an open-access article distributed under the terms of the Creative Commons Attribution License, which permits unrestricted use, distribution, and reproduction in any medium, provided the original author and source are credited. 
5. Ping Z, Ruan JF, Weng W, Tang Y (2020) Overexpression of SET and MYND domain-containing protein 2 (SMYD2) is associated with poor prognosis in pediatric $b$ lineage acute lymphoblastic leukemia. Leuk Lymphoma 61: 437-444.

6. Sakamoto LHT, Andrade RV, Felipe MSS, Motoyama AB, Silva FP, et al. (2014) SMYD2 is highly expressed in pediatric acute lymphoblastic leukemia and constitutes a bad prognostic factor. Leuk Res 38: 496-502.

7. Xin Y, Jiang XJ, Fang ZM (2019) HSistone methyltransferase SMYD2: Ubiquitous regulator of disease. Clinical Epigenetics 11: 112.
8. Edoardo F, Manoni E, Ferroni C, Del Rio A, Bartolini M (2019) Small-molecule inhibitors of lysine methyltransferases SMYD2 and SMYD3: Current trends. Future Medicinal Chemistry 11: 901-921.

9. Teresa R-T (2021) The SMYD family proteins in immunology: An update of their obvious and non-obvious relations with the immune system. Heliyon 7: e07387. 